\title{
Phase-field modelling of microstructural evolution in primary crystallization
}

\author{
Pere Bruna ${ }^{1,3, *}$, Eloi Pineda ${ }^{2,4}$, Daniel Crespo ${ }^{1,4}$ \\ ${ }^{1}$ Departament de Física Aplicada, EPSC, and ${ }^{2}$ Departament de Física i Enginyeria Nuclear, ESAB \\ Universitat Politècnica de Catalunya, Avda. del Canal Olímpic s/n, 08860 Castelldefels, Spain \\ ${ }^{3}$ Centre de Recerca en Nanoengineyeria and ${ }^{4}$ Centre de Recerca de l'Aeronutica i de l'Espai, UPC \\ *Pere.Bruna@upc.edu
}

Correspoding author: Daniel Crespo, Daniel.Crespo@upc.edu

\begin{abstract}
One of the main routes to obtain nanostructured materials is through the primary crystallization of metallic glasses. In such transformations, crystallites with a different composition than the amorphous precursor grow with a diffusion-controlled regime. Particle growth is slowed and eventually halted by the impingement between the concentration gradients of surrounding particles. Primary crystallization kinetics is not well described by the KJMA equation, and this fact was generally ascribed to both the soft-impingement effect and the non-random nucleation. However, recent phase-field simulations showed that the underlying physical reason is the change in the local diffusion properties of the amorphous precursor due to the variation of the composition during the transformation. The kinetics of primary crystallization is thus well described by considering a diffusion coefficient of the slowest diffusing species dependent on the local concentration. The nanostructure developed in such transformations is a key point to explain the macroscopic properties of these materials. In this work the grain size distributions obtained in realistic phase-field simulations of transformations with continuous nucleation and both constant and variable diffusion coefficient are presented. The obtained distributions are analyzed and the physical mechanisms responsible of their different features are recognized.
\end{abstract}

PACS codes: 81.10.Aj, 64.70.Kb, 81.30.Hd, 66.30.Pa, 07.05.Tp 
Keywords: nucleation and growth transformations, crystallization kinetics, microstructure development, diffusion controlled growth, phase-field modelling

\section{Introduction}

The kinetics of primary crystallizations has been for a long time a subject of controversy due to the unexpected delays observed between calorimetric data and the predictions of the different kinetic models used to describe it. Some kinetic models describe primary crystallization by means of a mean-field reduction of the crystalline growth rate as the transformation proceeds [1]. Such a reduction is commonly associated with a decrease of the concentration gradient surrounding the crystallites due to the overlap of the concentration fields of neighbouring particles: the soft-impingement (SI) effect. Other models account for the SI through geometrical considerations concerning the distribution of the transformed phase and the surrounding stabilized matrix regions [2]. However, these models do not seem to be able to fully explain the kinetics of such transformations. Recent realistic phase-field simulations [3][4] show that the observed experimental kinetics cannot be reproduced without considering the local change in the chemical composition of the alloy and the corresponding alteration of the atomic transport properties, i.e. the local diffusion coefficient.

The modification in the observed kinetics must influence the microstructure developed along the primary crystallization. In this work we show the microstructure obtained in the phase-field simulations, both with constant and concentration dependent diffusion coefficient, to confirm the effect of the change in the transport properties in the final microstructure.

\section{Primary crystallization models}

Primary transformations have been usually modelled as diffusion controlled growth processes in the framework of the Kolgomorov, Johnson-Mehl and Avrami (KJMA) model, though the SI effect does not fulfill the conditions of use of the KJMA equation [5].

Primary crystallizations end before occupying all the available space due to the stabilization of the 
untransformed phase that occurs simultaneously to the crystallization process itself; this stabilization also reduces, and eventually inhibits, the nucleation of new grains close to the existing grains, giving rise to what is generally known as non-random nucleation (NRN) [6]. It is common in the literature to describe the kinetics with a modified KJMA model, by normalizing the actual and the extended transformed fractions by the supersaturation value [2], that in these transformations is equal to the final crystallized fraction. We call this modification the normalized Avrami (NA) model, which is equivalent to a local stabilization of the untransformed matrix surrounding the crystallites due to its geometrical interference, hence modifying in some regions the growth kinetics. However, this model does not reproduce the complex behaviour of the Avrami exponent found in primary crystallization of metallic glasses, namely a variation of the Avrami exponent $n$ from values corresponding to either diffusion or interface-controlled growth at the initial stages of the transformation to values of $n<1$ at the final stages. In order to account for this experimental behaviour, Mora et al. proposed a further modification of the NA formulation, and introduced the variation of the kinetic parameters as a function of the transformed fraction through a mean-field (MF) model [1]. This model reproduces the experimental behaviour of the Avrami exponents all along the primary crystallization. Afterwards, the authors performed realistic phase field simulations that included the soft impingement effect to check the physical ground of the MF model [3]. Unexpectedly, these simulations did not agree with the MF model - and thus with the available experimental data - but were in full agreement with the normalized KJMA kinetics. It was also exposed that the MF model, although being able to reproduce the experimental data, is taking into account the SI effect twice: considering the stabilization of the untransformed matrix as a local geometrical effect and also as a global effect through the MF. Hence, the SI effect may not be the sole responsible of the slowing down in the kinetics observed in primary crystallization.

The above description implicitly assumes that the transport properties of the untransformed phase do not change along the transformation. However, the viscosity of a supercooled liquid changes several orders of magnitude near the glass transition temperature $\mathrm{T}_{\mathrm{g}}$. In this supercooled region, either temperature or compositional changes are expected to induce large variations of the viscosity and hence of the atomic transport 
properties of the glass. Primary crystallization occurs in a deeply supercooled liquid in metallic glasses. In many cases the crystallization temperature $T_{x}$ is not far over $T_{g}$. The compositional changes occurring in the glassy matrix during primary crystallization are then expected to influence the atomic transport properties and hence the crystallization kinetics.

In order to check this effect, a 2D PF model was used to simulate the transformation of a many-particle system without anisotropy in a diffusion controlled regime and with periodic boundary conditions [3]. The kinetics is given by the evolution of both the concentration $-c-$ and the phase $-\phi$ - variables, given by

$$
\begin{aligned}
& \frac{\varepsilon^{2}}{m} \frac{\partial \phi}{\partial t}=\phi(1-\phi)\left(\phi-\frac{1}{2}+30 \varepsilon \beta \gamma c \phi(1-\phi)\right)+\varepsilon^{2} \Delta \phi \\
& \frac{\partial c}{\partial t}+\frac{1}{\gamma}\left(30 \phi^{2}-60 \phi^{3}+30 \phi^{4}\right) \frac{\partial \phi}{\partial t}=\vec{\nabla}\left(\frac{D(c)}{D_{0}} \vec{\nabla} c\right)
\end{aligned}
$$

where $\varepsilon$ is the interfacial thickness, $\gamma$ is the initial supersaturation, $m$ is the interface kinetic coefficient and $\varepsilon \cdot \beta$ is the ratio between the interfacial width and the capillary length. All variables are conveniently nondimensionalized by a reference length $\omega$ and a reference time $\omega^{2} / D_{0}$.

The diffusion coefficient $D(c)$ was assumed to depend on the local concentration $c$. Small changes in $c$ can be responsible of important variations of $T_{g}$ in metallic glasses [7]. In this work we considered a linear relationship between $T_{g}$ and $c$. Considering $T_{x}$ well above $T_{g}$, the viscosity and the diffusion coefficient were related through a modified Stokes-Einstein relation valid for metallic glasses [8]

$$
\eta D^{2}=c t
$$

For the temperature dependence of the viscosity, a Vogel-Fulcher-Tamman (VFT) equation was considered. With these requirements, $D(c)$ can be written as:

$$
D(c)=K \exp \left(-\frac{H}{K_{B} T}\right) \exp \left(-\frac{B_{0}(c)}{T-T_{0}(c)}\right)
$$

where $H$ is the activation energy for the diffusion process, $K_{B}$ is the Boltzmann constant, $B_{0}$ is the VFT 
activation temperature and $T_{0}$ is the temperature at which viscosity would become infinite Both, $B_{0}$ and $T_{0}$, can be compositional dependent. For the sake of simplicity, it will be assumed that this dependence is solely through $T_{0}$ and that is analogous to the dependence of the glass transition temperature on the composition. Thus, the diffusion coefficient can be written as:

$$
D(c)=K^{\prime} \exp (-g(c))
$$

where $K^{\prime}$ is a constant in an isothermal process and the particular form of $g(c)$ will depend on the particular metallic glass under study. In this work, the used $g(c)$ is described in [4].

The Non-random nucleation effect cited above was also introduced in the phase-field model following [9]. A constant amount of randomly chosen cells were selected as embryos at each time step, and the nucleation probability was given by

$$
I(c)=I_{0} \exp \left\{-Q\left[\ln ^{-2}\left(c / c_{l}\right)-\ln ^{-2}\left(c_{0} / c_{l}\right)\right]\right\}
$$

where $c_{l}$ is the equilibrium concentration in the matrix and $Q$ accounts for the intensity of the NRN effect.

Simulations were performed in a matrix of size $1000 \times 1000$ with a supersaturation of 0.5 . Adequate statistics was obtained by averaging 10 simulations in each case.

\section{Results and conclusions}

Results obtained by phase-field model simulations will be labelled as DCT or DVAR when obtained with constant or variable - concentration dependent - diffusion coefficient, respectively. These results will be compared with the MF model described in [1], as it shows excellent agreement with experimental data and allows a much easier numerical treatment.

Figures 1 and 2 show the time evolution of the transformed fraction and of the Avrami exponent respectively, in the DCT and DVAR simulations compared to the MF model. The agreement is much better by using a variable diffusion coefficient, which, at the same time, gives a much better description of the underlying physical phenomena that control the growth process. 
Figure 3 shows the evolution of the average grain size and the corresponding standard deviation for both cases. The average grain size is considerably smaller in the DVAR than in the DCT case, from $25 \%$ at the beginning to $35 \%$ at the end of the transformation. Similar figures are obtained when considering the standard deviation ( $28 \%$ to $20 \%)$.

Figure 4 shows three snapshots of the grain size distribution in both cases, corresponding to transformed fractions of $0.1,0.3$ and 0.5 respectively. The smaller grain size in the DVAR case is again observed, and the maximum grain size shows a similar behaviour than the average grain size, about $30 \%$ smaller. The grain density is larger in the DVAR than in the DCT case, though the shape of the distribution is quite similar.

Figure 5 shows a snapshot of the final grain size distribution in one simulation representative of each case. The growth interference through the diffusion gradients of concentration is reflected in the irregular shape of a large number of grains. The higher density and smaller grain size obtained in the DVAR case are clearly seen at the naked eye. The apparent large scale structure of grain agglomerates is thought to be a visual artefact, not corresponding to any actual physical feature.

The high grain density found in the DVAR case can be explained taking into account the slowing down in the kinetics caused by the changes in the atomic transport properties of the amorphous matrix. As the grains grow more slowly, the stabilization of the matrix is delayed with respect to the DCT case, thus making possible the nucleation of more grains. In consequence, as the density of grains increases the interaction between them also increases, thus resulting in a distribution of grains of smaller size. Therefore, while the soft-impingement effect defines the shape of the grains, the non-random nucleation sets the density and the average size of the grains.

Summarizing, a realistic simulation of the primary crystallization process agrees with the available experimental data only when including the effect of the concentration of the diffusion species in the local transport properties. As a consequence, the developed microstructure shows higher grain density and finer grain size, about $30 \%$ smaller than the value obtained by considering a constant diffusion coefficient.

It is worth to note that the true physical process is three-dimensional, while the microstructural results presented here are two-dimensional. Three-dimensional computations are in course, and are expected to 
confirm the results presented here.

This work was funded by CICYT, MAT2007-60087 and Generalitat de Catalunya, 2005SGR00535 and 2005SGR00201.

\section{References}

[1] M.T. Clavaguera-Mora, N. Clavaguera, D. Crespo and T. Pradell.: Prog. Mater. Sci. 47, 559 (2002)

[2] M.P. Shepilov, J. Non-cryst. Solids 208, 64 (1996)

[3] P. Bruna, D. Crespo, R. González-Cinca, E. Pineda, J. Appl. Phys. 100 (2006) 054907.

[4] P. Bruna, E. Pineda, D. Crespo, J. Non-Cryst. Solids 353. (2007) 1002-1004.

[5] J.W. Christian, The theory of Transformations in Metals and Alloys, Pergamon Press, Oxford, (1974).

[6] E. Pineda, T. Pradell and D.Crespo, Philos. Mag. A 82, 107 (2002)

[7] M. Mao, Z. Altounian and D.H. Ryan, J. Non-cryst. Solids 205-207, 476 (1996)

[8] P.A. Duine, J. Sietsma and A. van der Beukel, Phys. Rev. B 48, 6957 (1993)

[9] J.D. Gunton, J. Stat. Phys. 95, 903 (1999) 


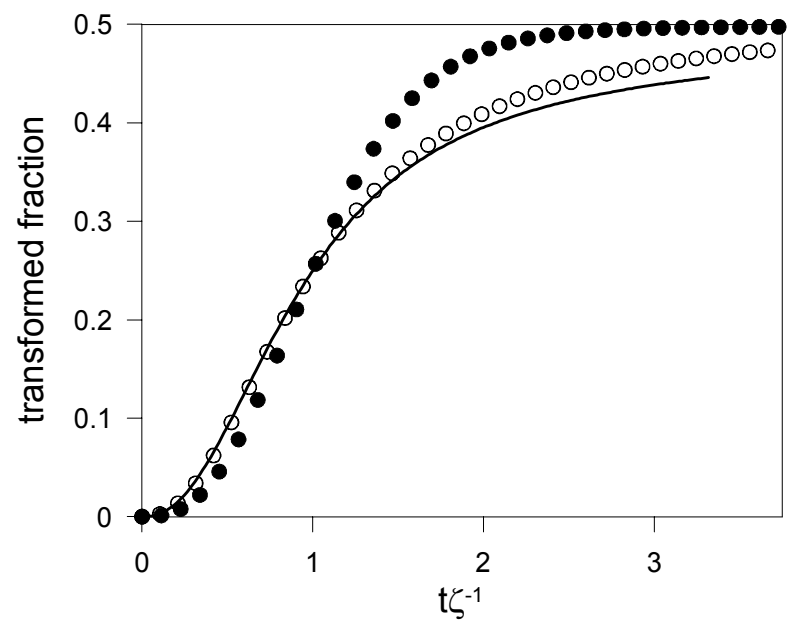

Figure 1 Transformed fraction versus time computed by phase field with constant (solid symbols) and variable (empty symbols) diffusion coefficient, compared to the model by Mora et al. [1] (continuous line). 


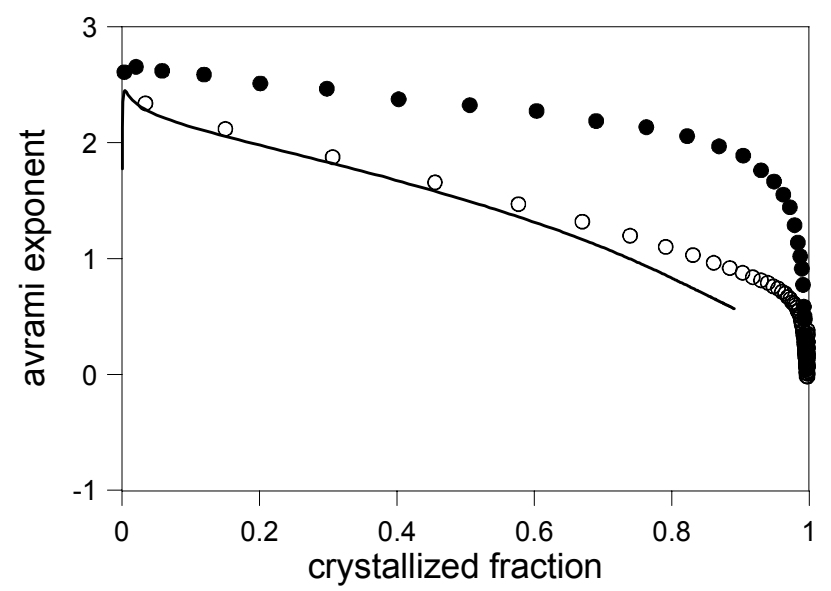

Figure 2 Avrami exponent versus transformed fraction. Same captions than in Figure 1. 


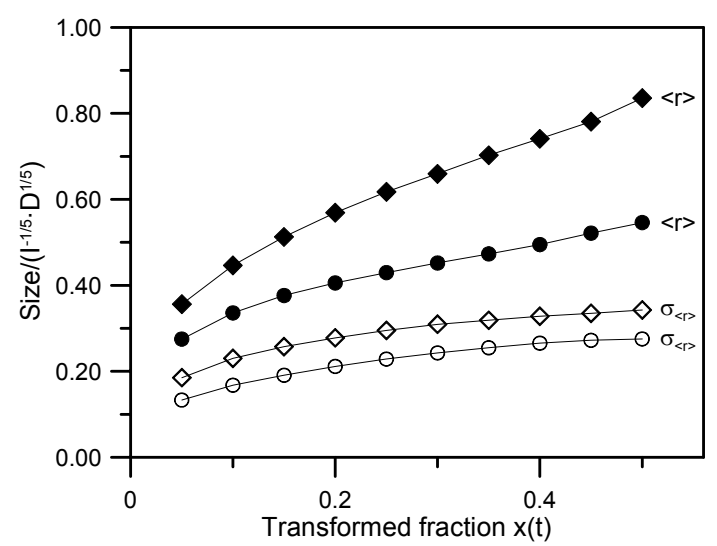

Figure 3 Average grain size (solid symbols) and standard deviation (open symbols) along the transformation computed by phase-field with constant (diamonds) and variable (circles) diffusion coefficient. 

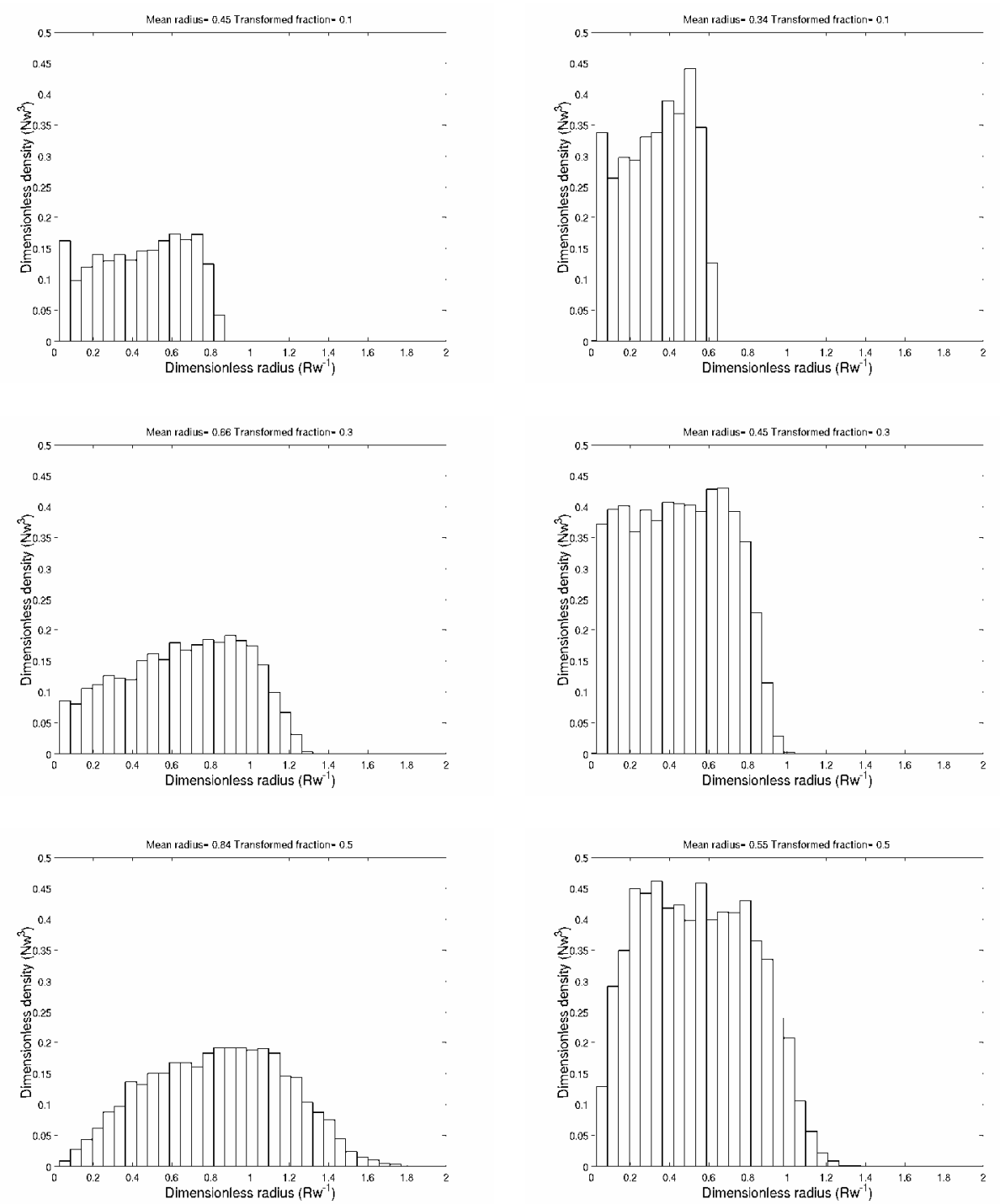

Figure 4 Grain size distribution at three different values of the transformed fraction 0.1 (top), 0.3 (centre) and 0.5 (bottom), computed with constant (left) and concentration dependent (right) diffusion coefficient. The concentration dependent diffusion coefficient simulation gives rise to a higher grain density of lower size. 

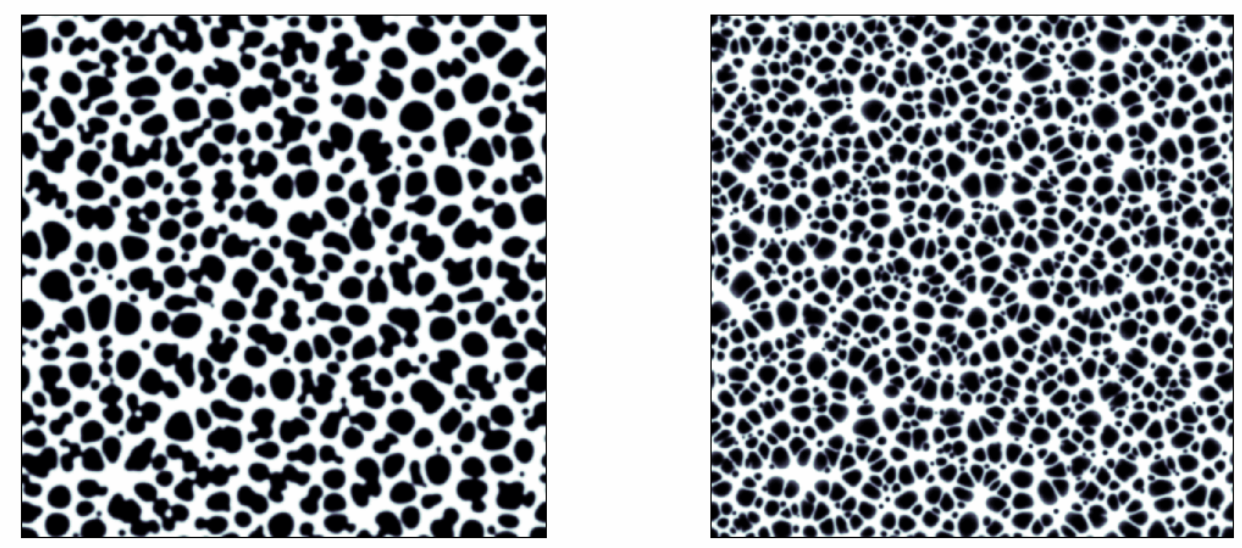

Figure 5 Final microstructure as simulated with constant (left) and concentration dependent (right) diffusion coefficient (right). Note the smaller grain size in the second case. 